\title{
Dacriocistorrinostomia endoscópica endonasal em casos de epífora com vias lacrimais pérvias
}

\author{
Endoscopic endonasal dacryocystorhinostomy in epiphora and patent lacrimal system
}

\author{
Denis Knijnik ${ }^{1}$ \\ Viviane Neutzling Uebel ${ }^{2}$ \\ Roberta Silveira Santos ${ }^{2}$
}

${ }^{1}$ Oftalmologista, instrutor de plástica ocular.

${ }^{2}$ Aluna do Curso de Especialização em Oftalmologia do Instituto Ivo Corrêa Meyer, Porto Alegre.

Endereço para correspondência: Rua Cel. Bordini 830 conj. 303 - Porto Alegre (RS) CEP 90440-003

E-mail: knijnik@hotmail.com

Recebido para publicação em 18.12.2001

Aceite para publicação em 12.06.2002

Nota Editorial: Pela análise deste trabalho e por sua anuência na divulgação desta nota, agradecemos à Dra. Suzana Matayoshi.

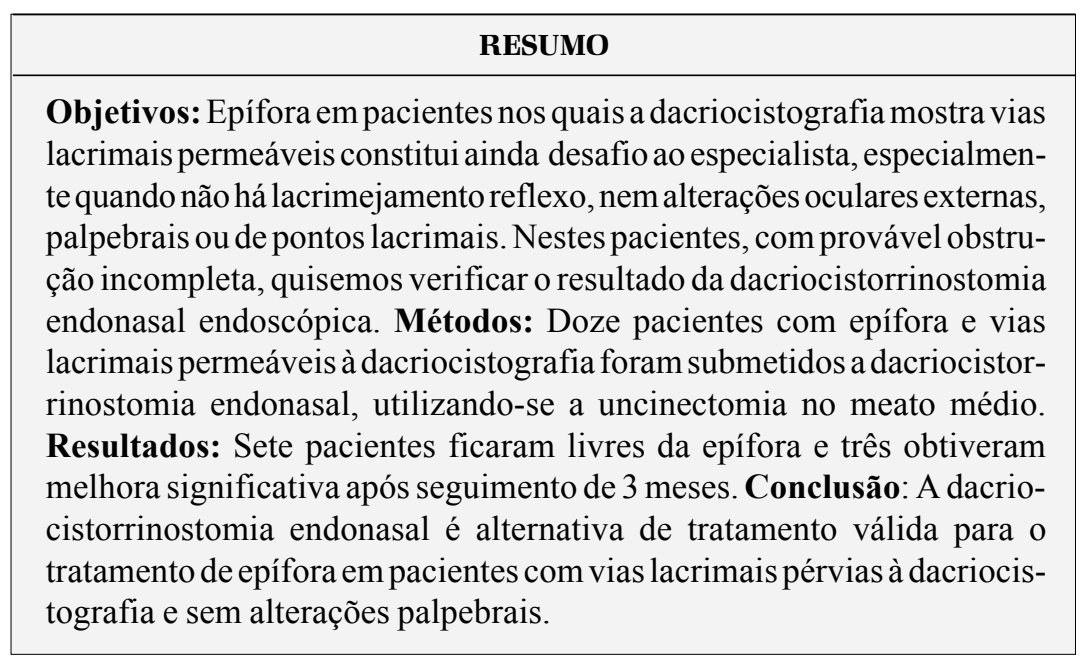

Descritores: Dacriocistorrinostomia; Doenças do aparelho lacrimal; Obstrução dos ductos lacrimais/cirurgia; Ducto nasolacrimal/cirurgia; Procedimentos cirúrgicos minimamente invasivos

\section{INTRODUÇÃ̃O}

Constitui ainda um desafio o tratamento de epífora crônica em pacientes com passagem de contraste à dacriocistografia e sem alterações nos pontos lacrimais ou pálpebras. Pacientes com este chamado "bloqueio funcional"(1) tem sido tratados de diversas formas (medicamentos, sondagens, intubação canalicular com silicone, tarsal "strip")(2), mas apenas uma minoria destes pacientes são submetidos a dacriocistorrinostomia.

No bloqueio funcional, a obstrução anatômica está incompleta ${ }^{(3)}$. Estudos histológicos tentam explicar o bloqueio funcional e o bloqueio completo (dacrioestenose adquirida) como sendo um mesmo processo patológico em estádios distintos de intensidade. No início do processo, a reação hiperêmica e o edema do canal ainda deixam passar, pela pressão da seringa, o líquido de irrigação ou contraste. Ainda caracterizando um bloqueio funcional, o processo avança com modificações estruturais causadas por dacriocistite crônica, de modo a produzir um segmento não funcionante no canal. Neste último caso, a obstrução ainda é incompleta, mas não há transporte lacrimal no segmento não funcionante ${ }^{(4)}$.

O’Donnell ${ }^{(5)}$ referiu excelentes resultados no tratamento da obstrução funcional através do mesmo tratamento utilizado para a obstrução completa, isto é, a dacriocistorrinostomia por via externa.

A dacriocistorrinostomia por via endonasal tem se afirmado como uma opção viável para a correção da dacrioestenose adquirida e formas complexas da congênita ${ }^{(6)}$. O objetivo deste trabalho foi verificar se a via endonasal 
também é aplicável ao tratamento dos pacientes com epífora que tem vias lacrimais pérvias.

\section{MÉTODOS}

Doze pacientes com epífora crônica e vias lacrimais permeáveis foram tratados com dacriocistorrinostomia endonasal de agosto de 1999 a junho de 2001. Fizeram parte deste estudo os pacientes nos quais a dacriocistografia mostrou passagem de contraste e que tiveram exame normal no que se refere a doenças externas, pontos lacrimais e posição e elasticidade da pálpebra.

Após termo de consentimento e vasoconstrição tópica endonasal, empregou-se anestesia local com sedação ou anestesia geral. A técnica utilizada foi a dacriocistorrinostomia endoscópica endonasal com uncinectomia: um iluminador de retina foi introduzido no canalículo superior e, por transiluminação, a localização do saco lacrimal foi confirmada com endoscopia nasal. As ópticas utilizadas foram as de $0^{\circ}$ e $70^{\circ}$. No meato médio, o processo uncinado e a parede óssea imediatamente anterior a ele foram removidos. A mucosa nasal anterior à raiz do corneto médio foi cauterizada. O saco lacrimal foi incisado e sua parede medial parcialmente removida após identificação do mesmo após introdução de uma sonda lacrimal no canalículo superior e distensão da parede medial.

A via lacrimal em 4 pacientes foi intubada com silicone que permaneceu por um período não superior a 6 semanas.

O seguimento pós-operatório foi de 3 meses. Os resultados basearam-se na avaliação dos sintomas pelos pacientes. Bom resultado foi definido como ausência ou melhora da epífora ao final dos 3 meses.

\section{RESULTADOS}

A idade dos pacientes variou de 7 a 88 anos, com média de 63 anos. Sugerindo alterações na drenagem lacrimal, a dacriocistografia de alguns destes pacientes apresentava dilatação do saco lacrimal ou estreitamentos discretos do canal nasolacrimal, porém em todos havia passagem do contraste para a fossa nasal.

A Tabela mostra que dos 12 pacientes com epífora e vias lacrimais pérvias submetidos à dacriocistorrinostomia endoscópica endonasal, 10 pacientes (83\%) obtiveram bom resultado. Destes, 7 pacientes ficaram livres da epífora e 3 pacientes obtiveram melhora significativa. Apenas 2 pacientes não melhoraram.

\section{DISCUSSÃO}

Obstruções completas do sistema lacrimal, distais aos canalículos, tem sido tratadas com excelentes resultados pela dacriocistorrinostomia, tanto por via externa como por via endonasal. Para estas obstruções, em virtude dos excelentes resultados, as dúvidas quanto à escolha do tratamento limi-

\begin{tabular}{|cccc|}
\hline \multicolumn{4}{|c|}{$\begin{array}{c}\text { Tabela. } \\
\text { Dados dos pacientes com epífora e vias lacrimais } \\
\text { pérvias submetidos a DCR endonasal }\end{array}$} \\
Idade & Sexo & Silicone & \multicolumn{1}{c|}{ Resultado } \\
7 & M & 3 sem. & bom (livre da epífora) \\
44 & F & não & bom (melhor da epífora) \\
56 & M & 4 sem. & insatisfatório \\
61 & M & não & bom (livre da epífora) \\
65 & F & não & bom (livre da epífora) \\
68 & F & 6 sem. & bom (livre da epífora) \\
69 & F & não & bom (melhor da epífora) \\
72 & M & não & bom (livre da epífora) \\
72 & F & não & insatisfatório \\
74 & M & 4 sem. & bom (livre da epífora) \\
80 & F & não & bom (melhor da epífora) \\
88 & F & não & bom (livre da epífora) \\
\hline
\end{tabular}

tam-se quase somente à via de abordagem e a detalhes de técnica. Entretanto, quando a irrigação ou a dacriocistografia demonstram passagem de líquido para a cavidade nasal, parece não existir um consenso sobre a melhor forma de tratar a epífora, especialmente quando não há história de dacriocistite nem alterações palpebrais.

Segundo um questionário enviado aos membros da American Society of Ophthalmic Plastic and Reconstructive Surgery ${ }^{(2)}$ sobre o tratamento de epífora sem obstrução mecânica ou falência da bomba lacrimal, $38 \%$ dos médicos não fariam nada, $28 \%$ usariam medicamentos, $15 \%$ entubariam com silicone, $6 \%$ fariam cirurgia para flacidez palpebral e apenas $4 \%$ fariam dacriocistorrinostomia.

Nós preferimos descrever os pacientes apenas como tendo sistema lacrimal permeável, sem recorrer a denominações que podem ser confusas. A terminologia para epífora com sistema lacrimal patente tem sido variada. Demorest e Milder utilizaram o termo "bloqueio funcional" em 1955 para referir-se a um sistema lacrimal em que a irrigação era normal mas a dacriocistografia mostrava retenção de contraste no saco lacrimal $^{(7)}$. Hurwitz et al. ${ }^{(1)}$ aplicaram o mesmo termo para aqueles com epífora e dacriocistograma normal. Já Rosenstock e Hurwitz preferiram o termo "disfunção fisiológica" e incluíram nele pacientes com flacidez palpebral e estenose do ponto lacrimal $^{(3)}$. Pacientes com estas últimas alterações não foram incluídos no presente trabalho.

A dacriocistografia é muito útil na localização e documentação da dacrioestenose. Mannor e Millman ${ }^{(8)}$ consideram o tamanho do saco lacrimal, conforme avaliado pela dacriocistografia pré-operatória, de valor prognóstico no resultado da dacriocistorrinostomia endonasal.

Na revisão da literatura nacional, não encontramos menção à uncinectomia como técnica utilizada para expor o saco lacrimal no decurso da dacriocistorrinostomia endonasal. A uncinectomia consiste em remover de início o processo unciforme no meato médio ${ }^{(9)}$. Esta técnica facilita e reduz o tempo cirúrgico, dispensando o uso de brocas para remover a parede óssea que separa o saco lacrimal da cavidade nasal. 
A dacriocistorrinostomia por via externa foi usada com sucesso no tratamento da epífora com sistema lacrimal pérvio e obstruções incompletas ${ }^{(3)}$. Para obstrução lacrimal completa, comparando-se a via externa com a via endoscópica, os resultados descritos na literatura tem sido um pouco melhores pela via externa ${ }^{(10)}$. O'Donnel refere excelentes resultados (94\%) com a DCR por via externa para epífora com sistema lacrimal patente $^{(5)}$. Embora um pouco inferiores aos dele, nossos bons resultados com dacriocistorrinostomia endonasal para o mesmo tipo de paciente indicam que estas duas técnicas diferentes de DCR são alternativas aceitáveis.

As vantagens da via endoscópica sobre a via externa incluem ausência de cicatriz na face e menos hemorragia. A via endoscópica, no entanto, apresenta também algumas desvantagens: a inspeção de anomalias do saco lacrimal é mais difícil, a anatomia nasal de alguns pacientes pode impedir a técnica e qualquer sangramento limita a visibilidade.

\section{CONCLUSÃO}

Epífora crônica, sem alterações palpebrais e com passagem de líquido pela pressão da seringa de irrigação ou dacriocistografia, pode ser sintoma de um bloqueio incompleto ou inicial. Concluímos que, para estes pacientes com vias lacrimais pérvias à dacriocistografia e sem alterações palpebrais, a dacriocistorrinostomia endoscópica endonasal é uma alternativa de tratamento válida.

\section{ABSTRACT}

Purpose: Epiphora in patients with a patent lacrimal system as demonstrated by dacryocystography is still a challenge to the specialist, mainly in the absence of reflex tearing, external ocular diseases or lid diseases. In these patients, with probable incom- plete obstructions, we wanted to evaluate the results of endoscopic endonasal dacryocystorhinostomy. Methods: Twelve of these patients were treated with endonasal dacryocystorhinostomy. Results: Seven of the patients became free of epiphora and three patients had their condition significantly improved. Conclusion: With no abnormalities of eyelids and puncta, endonasal endoscopic dacryocystorhinostomy is a valid treatment option for tearing with dacryocystographic patency.

Keywords: Dacryocystorhinostomy; Lacrimal apparatus diseases; Lacrimal duct obstruction/surgery; Nasolacrimal duct/surgery; Minimally invasive surgical procedures

\section{REFERÊNCIAS}

1. Hurwitz JJ, Maisey MN, Welham RAN. Quantitative lacrimal scintillography. Br J Ophthalmol 1975;59:313-22.

2. Conway ST. Evaluation and management of "functional" nasolacrimal blockage: results of a survey of the American Society of Ophthalmic Plastic and Reconstructive surgery. Ophthal Plast Reconstr Surg 1994;10:185-7.

3. Rosenstock T, Hurwitz J. Functional obstruction of the lacrimal drainage passages. Can J Ophthalmol 1982;17:249-55.

4. Paulsen FP, Thale AB, Maune S, Tillmann BN. New insights into the pathophysiology of primary acquired dacryostenosis. Ophthalmology 2001; 108:2329-36.

5. O'Donnell B, Shah R. Dacryocystorhinostomy for epiphora in the presence of a patent lacrimal system. Clin Experiment Ophthalmol 2001;29:27-9.

6. Woog JJ, Kennedy RH, Custer PL, Kaltreider SA, Meyer DR, Camara JG. Endonasal dacryocystorhinostomy: a report by the American Academy of Ophthalmology. Ophthalmology 2001;108:2369-77.

7. Demorest BH, Milder, B. Dacryocystography. II. The pathologic lacrimal apparatus. Arch Ophthalmol 1955;54:410-4.

8. Mannor GE, Millman AL. The prognostic value of preoperative dacryocystography in endoscopic intranasal dacryocystorhinostomy. Am J Ophthalmol 1992;113:134-7.

9. Fayet B, Racy E. L'unciformectomie est-elle la clef de la dacryocystorhynostomie par voie endonasale? J Fr Ophtalmol 2000;23:433-6.

10. Hartikainen J, Antila J, Varpula M, Puukka P, Seppa H, Grenman R. Prospective randomized comparison of endonasal endoscopic dacryocystorhinostomy and external dacryocystorhinostomy. Laryngoscope 1998;108:1861-6.

\title{
ABO ELETRÔNICO
}

\author{
Novo site
}

\section{Acesso: http://www.abonet.com.br}

